\title{
Erb's Palsy in a Newborn Child and Clinicoanatomical Approach
}

\author{
Debajani Deka \\ Silchar Medical College and Hospital, Silchar, Assam, India
}

Email address:

debajanideka31@gmail.com

\section{To cite this article:}

Debajani Deka. Erb's Palsy in a Newborn Child and Clinicoanatomical Approach. Biomedical Sciences. Vol. 7, No. 1, 2021 , pp. 17-19. doi: $10.11648 /$ j.bs.20210701.13

Received: January 5, 2021; Accepted: January 18, 2021; Published: February 2, 2021

\begin{abstract}
Erb's palsy is found in upper trunk of brachial plexus. C5, C6 roots join to from upper trunk. Each trunk again divides into anterior and posterior divisions. All trunks and divisions are found above the clavicle in the posterior triangle of neck. Branches from the upper trunk are suprascapular nerve and nerve to subclavius. Suprascapular nerve supplies supraspinatus and infraspinatus muscle along with it a articular trig to capsule of shoulder joint. Supraspinatus helps in initial $15^{\circ}$ abduction at shoulder joint. Infraspinatus acts as lateral rotator of shoulder joint and assists subscapularis and teres minor muscles to abduct at this joint when there is contraction of middle fibers of deltoid and supraspinatus. Some fibers of C5 also give contribution to accessory phrenic nerve. Lateral cord of brachial plexus giving- musculocutaneous nerve, lateral pectoral nerve, lateral root of median nerve. Musculocutaneous nerve supplies all flexor muscles of arm like biceps brachii, brachialis, coracobrachialis. Brachialis muscle flexes the elbow joint, coracobrachialis is a weak flexor of shoulder joint and biceps brachii is a strong supinator of forearm, flexor of elbow joint only when forearm is supinated. Injury occurs in the Erb's point found in the upper trunk of brachial plexus where there are emergence of six branches- C5 \& C6 nerve roots, suprascapular nerve, nerve to subclavius, anterior and posterior division of upper trunk of the plexus. In this palsy, the arm remains medially rotated and adducted at the shoulder joint, the elbow is extended or semiflexed, forearm is pronated, and the wrist semiflexed. In this case a newborn child was found unable to flex his elbow, and abducted the arm on the right side. The limb is medially rotated, adducted in shoulder joint, semiflexion at the elbow joint and forearm pronated and wrist semiflexed. The child was kept on artificial respiratory ventilation. Plain Chest X-ray done to see if any musculoskeletal abnormality is there but no positive findings found so far. Both the clavicles and all ribs were found intact. Both domes of diaphragm were moving normally. On the other hand left sided upper limb found fully flexed at the elbow joint, supinated at forearm, abducted externally rotated at the shoulder joint and wrist extended. Moros reflex on the right side was absent but on left side it was clearly present. So, there is asymmetrical Moros reflex.
\end{abstract}

Keywords: Erbs, Brachial, Clavicle, Fracture, Moros, Elbow, Injury, Arm

\section{Introduction}

Wilhelm Heinrich Erb is a eminent German neurologist. In 1883 he published his text 'Electrotherapeutics' where he mentioned that there is a circumscribed point midway between mastoid process and midclavicle called 'Erb's point' along the posterior border of sternocleidomastoid muscle. So, the term Erbs point is derived from his name. He tried experiments on contractions of arm muscles by giving transcutaneous electrical stimulations. [1] The other name for Erb's point is nervus punctum. This is the point of emergence of four sensory cutaneous nerves of cervical plexus and they are as follows-

1) lesser occipital nerve

2) greater auricular nerve

3) transverse cervical nerve

4) supraclavicular nerves

The spinal accessory nerve exits the posterior border of the sternocleidomastoid near this point. To provide superficial cutaneous anaesthesia around the periauricular region this point is preferred. $[11,13]$ There is another term Erb's palsy or Erb's Duchenne palsy which is a obstretic brachial plexus injury i,e. injury to the upper trunk of brachial plexus mainly, where there are emergence of six nerves -C5, C6 nerve roots, 
suprascapular nerves, nerve to subclavius anterior \& posterior division of brachial plexus. We can define mainly three pathological terms depending on degree of severity of injury to these nerves -neuropraxic, axonometric or neurometric injury accordingly. Neuropraxia is nerve compression injury which is reversible, axonometric injury is disruption of axon \& myelin sheath which takes months to heal with proper treatment and physiotherapy whereas neurometric injury is irreversible where there is avulsion of roots, axons and myelin sheath along with structures enervated by these nerves. It is $50 \%-60 \%$ common birthrelated brachial plexus injury. During traction of aftercoming head in some cases of difficult labour there may be stretch, rupture or avulsion of roots in cervical part of spinal cord leading to Erb's palsy. [2-4] Erb's palsy due to traumatic pervaginal delivery can be devided into: (1) upper Erb's palsy which involves injury to C5 \& C6 roots of spinal cord. Here the newborn is unable to abduct the shoulder, externally rotated the arm and flex the elbow. (2) Extended Erb's palsy involves C5, C6, C7 nerve roots and hence wrist drop is also seen; the new born presents with the "waiter's tip posture" where the shoulder is adducted, arm is internally rotated, the elbow is extended, and the wrist is flexed i.e. wrist drop. (3) Total palsy, all roots of the brachial plexus are involved C5, C6, C7, C8 as well as T1.. All these injuries neonate suffers from severe traction on neck. In case of cephalic presentation, there is usually a history of shoulder dystocia because of fetal macrosomia. In breach presentation, there is usually a difficulty of delivery of the "after coming head". In Cesarean section deliveries, through the uterine incisions there is excessive manipulations of head and shoulder causing injury. [5] Ernst Moro in 1918 first coined the term Moro Reflex. This reflex is a primitive reflex seen in neonate immediately after birth being seen in some preterm infants at 25 weeks of Postconceptional age (PCA) and in the majority by 30 weeks of PCA. It usually disappears by 2-3 months of age and complete disappearance by 6 months of age after birth. Persistence of this reflex beyond 6 months is considered abnormal. The absence of the response during the neonatal period and early infancy suggests a compromised condition or disorder including birth injury, severe birth asphyxia, intracranial hemorrhage, infection, brain malformation, general muscular weakness of any cause, and Cerebral palsy of the spastic type. Asymmetry of the response is usually a sign of local injury like damage to a peripheral nerve or cervical spinal cord or a unilateral clavicle fracture. [14] The diaphragm is the primary muscle of respiration and its weakness can interfere with normal respiratory functions. [15] Left anterior occipitoiliac presentation leads to excessive traction on right neck during vaginal delivery as right shoulder remains under the pubis. Because of this this type of injury is most prevalent on right side. In $4 \%$ of cases injury can be bilateral. [16] Nerve repair in brachial plexus injury involves resection of neuroma or nerve grafting usually by sural nerve where damaged section of nerve is rejected and nerve graft is placed connecting the proximal and distal ends of the rejected nerve. [17]

\section{Case Report}

In this case, a newborn child was found unable to flex his elbow, and abducted the arm on the right side. The limb is medially rotated, adducted in shoulder joint, semiflexion at the elbow joint and forearm pronated and wrist semiflexed. The child was kept on artificial respiratory ventilation. Plain Chest $\mathrm{X}$-ray done to see if any musculoskeletal abnormality is there but no positive findings found so far. Both the clavicles and all ribs were found intact. Both domes of diaphragm were moving normally. On the other hand left sided upper limb foundfully flexed at the elbow joint, supinated at forearm, abducted externally rotated at the shoulder jointand wrist extended. Moors reflex on the right side was absent but on left side it was clearly present. So, there is asymmetrical Moors reflex. From a clinic anatomical perspective we can conclude that there must be avulsion injury to the cervical nerve roots on right side during delivery in a difficult labour like pervaginal delivery of a macrsomic baby, aftercoming head in breech presentation, shoulder dystocia etc. It may be also found in Cesarean section during excessive manipulation of the baby. Here this is a case of total Erb's palsy involving $\mathrm{C} 5, \mathrm{C} 6, \mathrm{C} 7, \mathrm{C} 8, \mathrm{~T} 1$ roots because the elbow is somehow found to be extended. In upper Erb's palsy and extended Erb's palsy elbow is found in extended position. Due to avulsion of $\mathrm{C} 5$ nerve root there is decrease impulse transmission to accessory phrenic nerve (motor nerve fibers to muscles of diaphragm) nerve of respiration leading to difficult ventilation and because of which the child was kept on artificial ventilation. The chest on right side is also found elevated.

\section{Discussion}

E. Christopher W. and Yasir A. Khalil mentioned that moros reflex is an infantile reflex. The normal response is characterized by sudden, symmetrical, extension/ abduction movements of the arms, and extension of the fingers along with extension of neck and spine followed by adduction of the shoulders and flexion of the arm. A normal Moro is commonly a very symmetrical response. It becomes asymmetrical in the presence of brachial palsy and in the rare cases of neonatal hemiplegia or other pathological states like intracranial hemorrhage, cerebral palsy of spastic type, birth asphyxia. Fractures of the clavicle or homers or injury to the shoulder joint are also common causes of asymmetry. [6]

Fatima, Sudan Gomez, Julian et. al, in 2019 mentioned that in upper brachial plexus palsy (C5-C6) is characterized by impaired abduction and external rotation at the shoulder and flexion of elbow joint, while hand function is preserved. Also known as Dejerine-Klumpke syndrome, lower brachial plexus palsy (C7-T1) impairs hand and wrist function. In the case of a complete brachial plexus palsy (C5-T1), the function of the entire arm is impaired, presenting with a completely flaccid arm without sensitivity, and sometimes with ocular impairment. This combination of symptoms is known as Horner's Syndrome [7].

S. Deepak, P. Aakash, S. Sweta et. al, in 2016 found that in 
Erb's palsy usually there is typically adduction and internal rotation at the shoulder joint. At the elbow joint, there is extension and pronation with flexion of wrist and fingers. The most commonly involved nerves include suprascapular nerve, musculocutaneous nerve, and the axillary nerve. The muscles which are affected include deltoid, infraspinatus, biceps, supinator, and brachioradialis muscles of the upper limb. $[8,9]$

Brachial plexus injury is mostly found in 1-4 per 1000 live births and $66 \%-92 \%$ of cases recover spontaneously before two months of age. In shoulder dystocia related injury it is always associated with ipsilateral phrenic nerve injury in $2 \%$ $5 \%$ of infants. This leads to ipsilateral diaphragmatic palsy and respiratory difficulty. Infant is kept under positive pressure ventilation, nasogastric intubation. Surgical interventions applied mainly diaphragmatic plication. [2, 10]

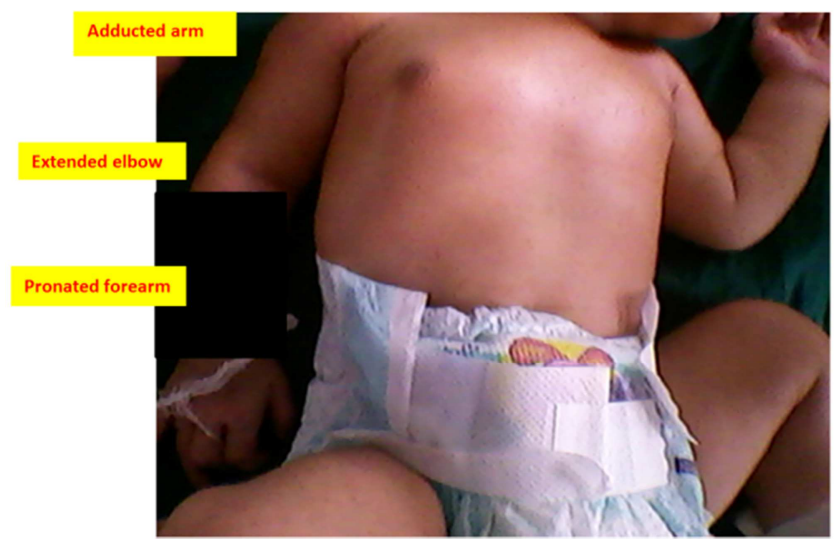

Figure 1. Erb's Palsy in a newborn child.

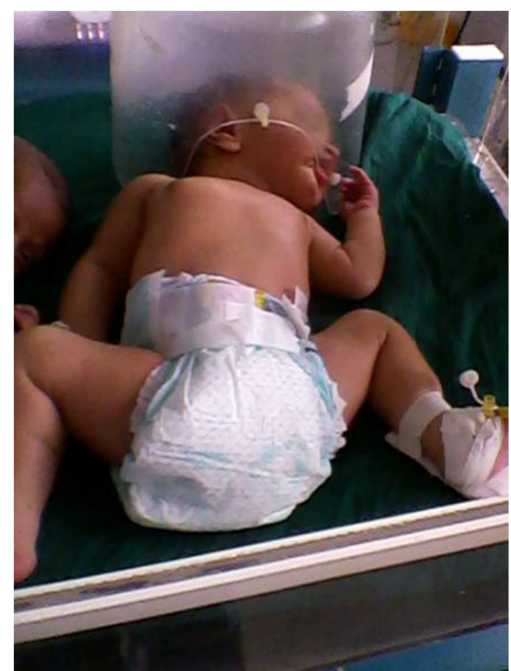

Figure 2. Newborn child on artificial respiratory ventilation.

\section{Conclusion}

From clinicoanatomical perspective, in this type of case we can come into a diagnosis of difficult respiration in a newborn infant only with the help of clinical examinations (primitive reflexes, respiratory rate, subcoatal recession) and few simple investigations (plain chest $\mathrm{x}$ ray, ultrasonography) when delivery is usually performed in an untrained hand.

\section{References}

[1] https://accessanesthesiology.mhmedical.com

[2] MR Thatte, A Hiremath, N Nayak, N Patel. Obstetric Brachial Plexus Palsy. Diagnosis and Management Strategy. Journal of Peripheral Nerve Surgery. Vol- 1, (1) 2017 pg: 2-9.

[3] Erb-Duchenne and Dejerine-Klumpke Palsies Information Page: National Institute of Neurological Disorders and Stroke (NINDS).

[4] Raducha JE, Cohen B, Blood T, Katarincic J. A review of brachial plexus birth palsy: Injury and rehabilitation. Rhode Island Medical Journal. 2017 Nov 1; 100 (11): 1721.

[5] Mohammad M. Al-Qattan \& Amel A. F. El-Sayed. A case of Klumpke's obstetric brachial plexus palsy following a Cesarean section. Clinical case report. 2016 Sep; 4 (9): 872-875.

[6] https://europepmc.org/article/med/31194330

[7] Fátima Frade, Juan Gómez-Salgad, Lia Jacobsohn and Fátima Florindo-Silva. Rehabilitation of Neonatal Brachial Plexus Palsy: Integrative Literature Review. J. Clin. Med. (2019) vol8 pg: 980.

[8] Deepak Sharma, Aakash Pandita, Sweta Shastri, Pradeep Kumar Sharma. Duchenne-Erb's palsy in newborn: Result of birth trauma Medical Journal of Dr. D. Y. Patil University March-April 2016 Vol 9 (2).

[9] Bannister LH, Berry MM, Collins P, et al. Gray's Anatomy, anatomical basis of anatomy and surgery. 38th ed. London: Churchill Livingstone;. p. 12661267.

[10] Audra J. Reiter, Yazan K. Rizeq, Benjamin T. Many, Jonathan C. Vacek, Fizan Abdullah, and Seth D. Goldstein. A Rare Case of Contralateral Diaphragm Paralysis following Birth Injury with Brachial Plexus Palsy: A Case Report and Review of the Literature. Case Reports in Pediatrics. vol-2020 pg: 1-6.

[11] Jaspreet Johal, Joe Iwanaga, Kevin Tubbs, Marios Loukas, Red J. Oskouion, R. Shane Tubbs. The Accessory Nerve: A Comprehensive Review of its Anatomy, Development, Variations, Landmarks and Clinical Considerations (2019) The anatomical record vol-302 pg: 620-629.

[12] M. C McGrath. The Vulcan Nerve Pinch - Cultural Iconography Anchors the Proposal of a Novel Manual Approach, the Bow-String Technique. Int J Complement Alt Med 2017, 5 (5): pg-1-8.

[13] Dr. Ian Bickle, Craig Hacking. Punctum nervosum. https://radiopaedia.org/articles/punctum-nervosum. 7 January 2021.

[14] Laxmi Kokatnur. ID and Mohan Rudrappa. Diaphragmatic Palsy. Diseases 2018, vol-6 (16). pg-1-14.

[15] A. Abid. Brachial plexus birth palsy: Management during the first year of life. Orthopaedics \& Traumatology: Surgery \& Research. vol-102 (2016) S125-S132.

[16] https://www.intechopen.com/books/treatment-of-brachialplexus-injuries/current-concept-in-the-management-ofbrachial-plexus-birth-palsy. 